\title{
CATION PERMEABILITY INDUCED BY TWO AROMATIC HEPTAENES, VACIDIN A AND CANDICIDIN D ON PHOSPHOLIPID UNILAMELLAR VESICLES
}

\author{
B. Cybulska and E. Borowski \\ Department of Pharmaceutical Technology and Biochemistry \\ Technical University, Gdansk, Poland \\ Y. Prigent and C. M. Gary-Bobo* \\ Service de Biophysique, Département de Biologie, CEN Saclay \\ 91191 Gif-Sur-Yvette Cedex, France
}

(Received for publication March 30, 1981)

\begin{abstract}
The cationic permeability induced by two aromatic heptaenes, vacidin A and candicidin D, has been studied on egg yolk L- $\alpha$-phosphatidylcholine single walled vesicles as a function of cholesterol and ergosterol concentration. For comparison amphotericin B and nystatin were also tested.

Vacidin A and candicidin D elicit cation permeability in both types of vesicles in the same concentration ranges and exhibit only quantitative differences in cholesterol and ergosterol vesicles. The active concentration range is of the same order of magnitude as the active concentration range of amphotericin B, at variance with what is obtained on biological cells. This difference is interpreted in term of mechanism of action of polyene on both biological and model membranes.
\end{abstract}

There are about one hundred known polyene macrolide antibiotics ${ }^{1)}$. Among them, the so called "aromatic heptaenes" are particularly promising.

These aromatic heptaenes, like the other large macrolide ring polyene antibiotics studied, induce permeability changes in sterol-containing cell membranes. However this action exhibits some particular features: The permeability change that they induce in eucaryotic microorganisms ${ }^{2 \sim 8)}$ and animal cells $^{2,7 \sim 11)}$ is specific for monovalent cation, and appears reversible to a large extent ${ }^{4,11,12)}$. Moreover as shown on red blood cells and on black lipidic films, this permeability exhibits a higher degree of intercationic selectivity ${ }^{7,14)}$. At variance with amphotericin B and nystatin, cholesterol-containing cells seem to be more sensitive to some of them than those containing ergosterol ${ }^{13}$. The feature which prompted the interest for these aromatic heptaenes is that their biological activity is about two to three orders of magnitude higher than non-aromatic heptaenes ${ }^{1,8,10,12)}$. Membrane effects of aromatic heptaenes have not been so well characterized, because, until recently, they were not available in a pure chemical form but only as complex mixtures of related compounds, and their chemical structure was not completely elucidated.

In this report data are presented concerning the permeability effect of two of these aromatic heptaenes, vacidin $\mathrm{A}$ and candicidin $\mathrm{D}$, studied on a model system made of egg yolk phosphatidyl choline single walled vesicles, as a function of cholesterol and ergosterol content. For comparison, data obtained on the same system and under the same experimental conditions with the now well-characterized amphotericin B and nystatin are also presented.

* To whom correspondence should be sent. 
Fig. 1. Molecular formula of vacidin A and candicidin D.

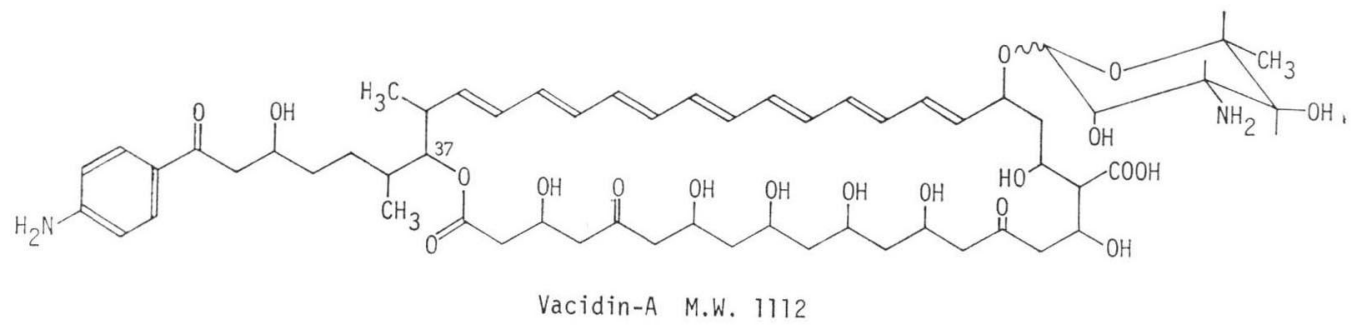

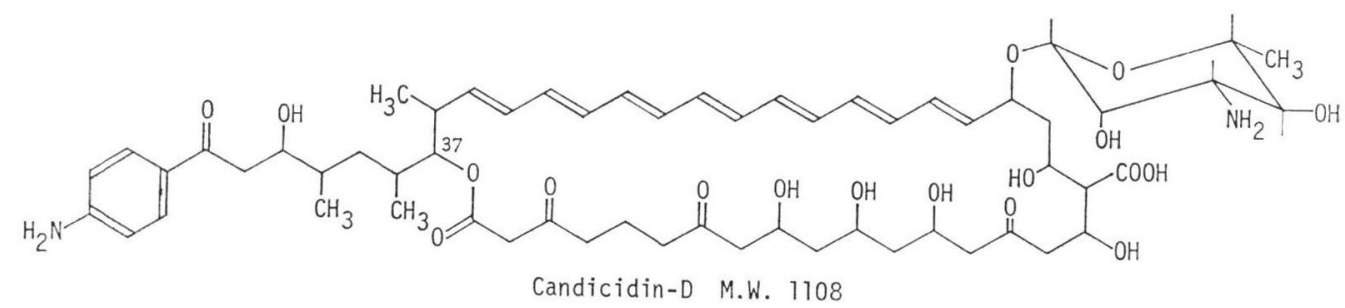

The chemical structures of vacidin $\mathrm{A}$ and candicidin $\mathrm{D}$ are presented in Fig. $1^{7)}$ : all members of the aromatic heptaene group are characterized by a $p$-aminophenyl residue attached to the macrolide ring in position 37 by a six carbon atoms aliphatic chain. Candicidin $\mathbf{D}$ and vacidin $\mathrm{A}$ have a cistrans heptaene chromophore ${ }^{\text {() }}$ as compared to the all-trans heptaene chromophore of amphotericin B.

\section{Materials and Methods}

L- $\alpha$-Phosphatidylcholine from egg-yolk was prepared according to Patel and Sparrow ${ }^{15)}$. Cholesterol was supplied by Fluka, ergosterol by Sigma, carbonyl cyanid- $\alpha$-trifluoromethoxyphenyl hydrazone (FCCP) by Boehringer. Amphotericin B and nystatin were Squibb products, whereas candicidin $\mathrm{D}$ and vacidin A were isolated and purified in the Department of Pharmaceutical Technology, Technical University, Gdansk, Poland.

Vesicles suspensions were prepared by dissolving weighted amounts of phospholipid and sterol in chloroform. After removal of the solvent $400 \mathrm{~mm}$ sodium phosphate $\mathrm{pH} 5.20(1 \mathrm{ml} / 30 \mu$ mole lipid) was added. The sample was sonicated under nitrogen stream at $4^{\circ} \mathrm{C}$. The vesicles suspension was dialysed at equilibrium against 500 volumes of isotonic sodium sulphate.

Proton efflux was measured using a pH-stat (Radiometer, Copenhagen) in the following way: $0.5 \mathrm{ml}$ of vesicles suspension ( $15 \mu$ mole lipids) was diluted with $3.5 \mathrm{ml}$ of dialysing buffer in the $\mathrm{pH}$-stat titrating vessel and equilibrated at $25^{\circ} \mathrm{C}$ under constant nitrogen stream. The $\mathrm{pH}$ was brought to 7.40 by addition of $30 \mathrm{~mm} \mathrm{NaOH}$ in isotonic sulphate. Then $10 \mu \mathrm{l}$ of a $10^{-2} \mathrm{M}$ FCCP solution in ethanol was added (final concentration $2.5 \times 10^{-5} \mathrm{M}$ ). Subsequently, the desired amount of polyene was added as microliter volumes of a freshly made $1 \mathrm{mg} / \mathrm{ml}$ solution in dimethyl formamide (DMF) using Hamilton syringes. The proton efflux was measured as the volume of $4 \mathrm{~mm} \mathrm{NaOH}$ solution in isotonic sodium sulphate required to maintain $\mathrm{pH} 7.40$. All experiments were carried out at $22^{\circ} \mathrm{C}$.

\section{Results}

Principle of the Method of Permeability Measurements

This comparative study of cation permeability induced by different polyene antibiotics is based upon the measurement of proton-cation exchange through the vesicle membrane: sonicated vesicles 
Fig. 2. Typical time course of an experiment.

The amount $(\mu \mathrm{I})$ of $4 \mathrm{~mm} \mathrm{NaOH}$ solution necessary to maintain $\mathrm{pH} 7.40$, is recorded as a function of time. Arrows indicate times of addition.

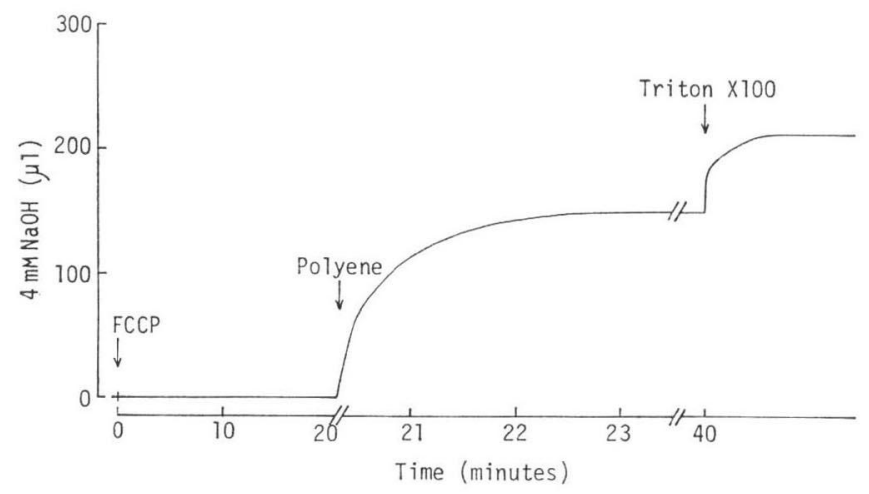

of egg-yolk phosphatidylcholine containing sterol or not, are rigorously impermeant to hydrophilic ions such as phosphate or sulphate and it has been shown previously ${ }^{18)}$ by ${ }^{31} \mathrm{P}$ NMR spectroscopy that vesicles prepared as described above are able to maintain more than a $4 \mathrm{pH}$ unit difference across their membrane during several hours or days depending upon lipid composition. In this system any cation movement across the vesicle bilayer can take place only by strictly electroneutral exchange. The addition of the proton carrier FCCP to vesicles submitted to a large $\Delta \mathrm{pH}$ does not result in any proton net flux and another path must be provided for the exchanging cation. In this condition, the permeability to cation induced by polyene antibiotics on vesicles submitted to a $\mathrm{pH}$ difference between intravesicular and external medium can be conveniently measured by monitoring the proton flux occurring in exchange through FCCP, provided this proton flux is not rate limiting. This condition has been proved easy to fulfil at least at the low or moderate polyene concentrations used in this study.

\section{Verification of the Experimental Conditions}

The typical course of an experiment is shown in Fig. 2. It has been shown, by preliminary experiments, that 1) in absence of any ionophore, the proton efflux is practically undetectable, amounting to about $0.1 \sim 1 \%$ of the total titratable protons in the sample per hour, depending upon lipid composition; 2) FCCP added alone is not able to promote any significant increase of this basic flux even at concentration as high as $10^{-3} \mathrm{M} ; 3$ ) the proton efflux mediated by FCCP in the presence of polyene is maximum and independent of FCCP concentration above $10^{-5} \mathrm{M}$; 4) the total amount of titratable proton in the vesicle sample was measured after Triton X-100 addition: the intravesicular volume calculated on this basis was routinely found equal to $1.2 \pm 0.2 \%$ of the volume of the $30 \mu \mathrm{mole} \mathrm{lipid} / \mathrm{ml}$ stock vesicles suspension. Under the influence of FCCP + polyene (in the highest concentration range studied) more than $95 \%$ of this amount was titratable; this confirms the fact already shown by ${ }^{31} \mathrm{P}$ NMR spectroscopy $^{16)}$ that most vesicles are unilamellar, since it may be considered that the polyenes studied here cannot reach the inner layers of multilayered vesicles ${ }^{17)}$. In these conditions ${ }^{18)}$, the number of vesicles in the sample (each being made of about 2,500 phospholipids molecules) can be calculated and the concentration of polyene expressed either in $\mu \mathrm{mole} / \mu \mathrm{mole}$ lipids, or in number of polyene molecules per vesicles. 
Fig. 3. Proton efflux (in $\%$ of total titratable proton) elicited in cholesterol (A) or ergosterol (B) containing vesicles under the influence of vacidin A: 1) 0.3 ,2) $0.65,3) 1.3$ and 4) $2.6 \times 10^{-3} \mu$ mole $/ \mu$ mole lipids.

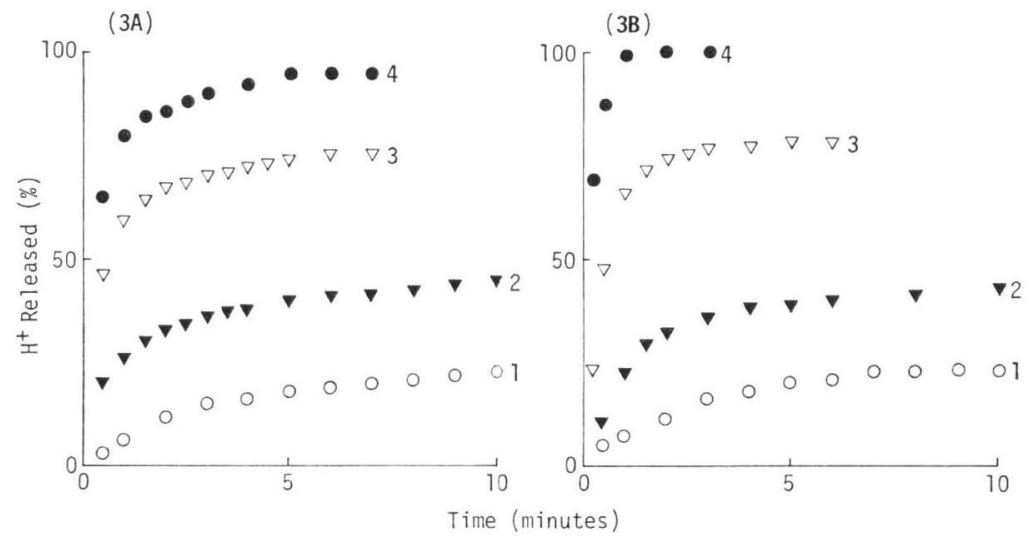

Permeability Induced by Vacidin A and Candicidin D

In Fig. 3 are given typical time courses of proton efflux from vesicles containing 20 mole $\%$ of cholesterol (Fig. 3A) and 20 mole \% of ergosterol (Fig. 3B) upon the addition of vacidin A at various concentrations. The proton efflux is expressed as the percentage of the total amount of titratable proton in the sample measured after Triton X-100 addition. Two main features are to be noted. First, the proton efflux which starts within 1 or 2 seconds after polyene addition develops rapidly and more so with increasing concentration. After 5 to 10 minutes at most, the amount of proton released reaches a plateau which remains stable with time (several hours). Second, the totality ( $100 \%$ ) of the titratable proton is released only at the highest concentration of vacidin A. The percentage released decreases with decreasing concentration. A new addition of vacidin A at the plateau promotes the release of more proton until $100 \%$ is reached (not shown on the figure).

It can be seen that comparable results are obtained in cholesterol and ergosterol-containing vesicles, the only significant difference being a slightly faster efflux rate in ergosterol-containing vesicles.

Qualitatively and quantitatively the results obtained with candicidin D are quite similar.

Fig. 4. Maximal proton release (in $\%$ of total titratable proton) as a function of concentration of cholesterol (A) and ergosterol (B) under the influence of vacidin A: 1) $0.3,2$ ) $0.65,3$ ) 1.3 and 4) $2.6 \times 10^{-3} \mu$ mole/ $\mu$ mole lipids.
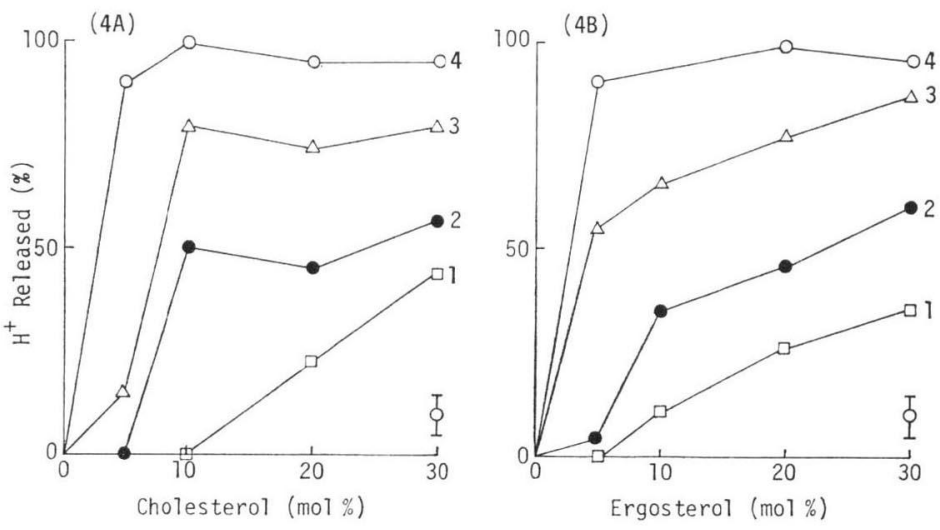
Fig. 5. Same as Fig. 4 but for candicidin D: 1) 0.4 , 2) 0.8 , 3) 1.6 and 4) $2.6 \times 10^{-3} \mu$ mole/ $\mu$ mole lipids.
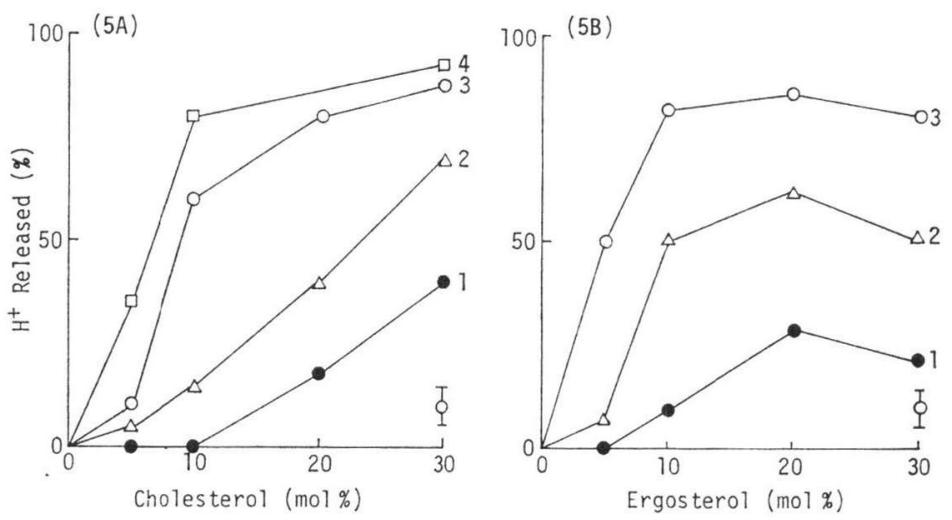

Table 1. Sensitivity to polyenes of cholesterol and ergosterol containing vesicles.

\begin{tabular}{l|c|c|c|c}
\hline \multicolumn{1}{|c|}{ Polyenes } & $\begin{array}{c}\text { Cholesterol } \\
(\mu \text { mole } / \mu \text { mole } \\
\text { lipids })\end{array}$ & $\begin{array}{c}\text { Number molecules } \\
\text { vesicles } \\
(10 \text { mole } \%)\end{array}$ & $\begin{array}{c}\text { Ergosterol } \\
(\mu \text { mole } / \mu \text { mole } \\
\text { lipids })\end{array}$ & $\begin{array}{c}\text { Number molecule/ } \\
\text { vesicles } \\
(10 \text { mole } \%)\end{array}$ \\
\hline Amphotericin B & $1 \times 10^{-3}$ & 2 & $2 \times 10^{-3}$ & 4 \\
Nystatin & $5 \times 10^{-3}$ & 10 & $4 \times 10^{-3}$ & 8 \\
Vacidin A & $2.5 \times 10^{-3}$ & 5 & $2.5 \times 10^{-3}$ & 5 \\
Candicidin D & $3.0 \times 10^{-3}$ & 6 & $2.5 \times 10^{-3}$ & 5 \\
\hline
\end{tabular}

In Figs. 4 and 5 are summarized the data obtained with different sterols concentrations, for vacidin $\mathrm{A}$ and candicidin $\mathrm{D}$ respectively. In these figures the percentage of titratable proton obtained at the plateau is plotted as a function of cholesterol (Figs. 4A and 5A) or ergosterol (Figs. 4B and 5B) concentration in mole \%.

First it can be seen that sterol-free vesicles are insensitive to both aromatic heptaenes. Second, the percentage of proton released increases with heptaene concentration (as already seen in Figs. 2 and 3) and sterol concentration, in both cases, but in a somewhat different way.

Vacidin A: increasing the cholesterol content beyond 10 mole per cent does not result in a greater efficiency of the heptaene. At the lowest vacidin A concentration, a significant proton release is obtained only beyond 10 mole $\%$ cholesterol and the maximum release is not yet reached with 30 mole $\%$ cholesterol. In ergosterol-containing vesicles the release increases with increasing sterol concentration.

Candicidin D: a maximum seems to be reached for 20 mole \% ergosterol, while a continuous increase is observed with cholesterol concentration.

\section{Permeability Induced by Amphotericin B and Nystatin}

In order to compare the action of the two aromatic heptaenes with the action of non-aromatic ones in the same experimental conditions, experiments have been carried out with amphotericin B and nystatin. The results obtained by the present method generally confirm the data already published in the literature concerning the sensitivity of ergosterol and cholesterol-containing vesicles ${ }^{19,20)}$ and the dependency upon sterol concentration. However, two particular results should be stressed: first, sterolfree vesicles appear to be sensitive to amphotericin B and significant proton release is obtained at concentration as low as $0.2 \times 10^{-3} \mu$ mole $/ \mu$ mole lipids. 
Fig. 6. Same as Fig. 3 but for nystatin: 1) $0.4,2) 0.8,3) 1.6$ and 4) $2.6 \times 10^{-3} \mu$ mole $/ \mu$ mole lipids.

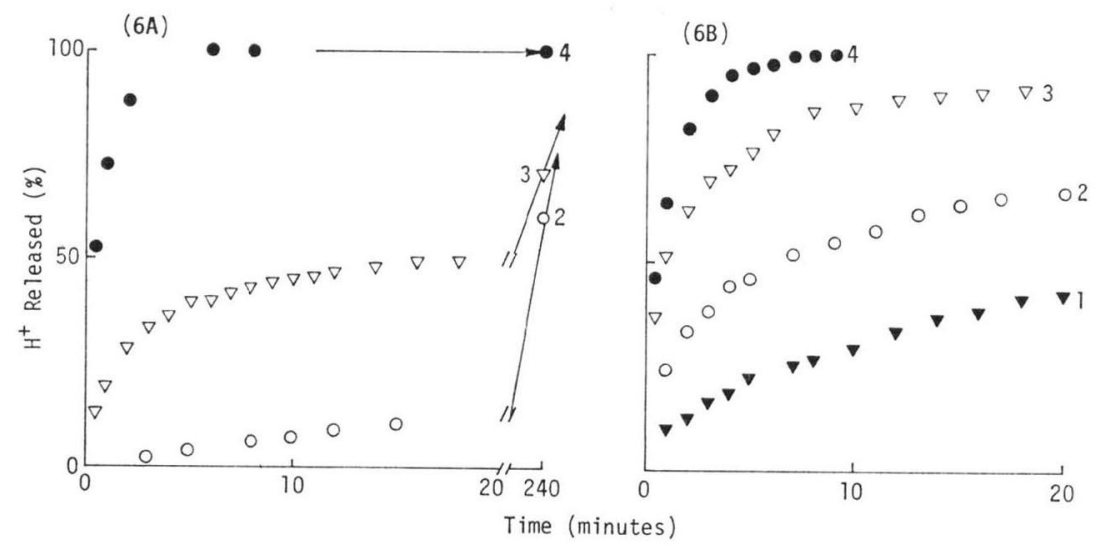

Second, vesicles containing sterol (either cholesterol or ergosterol) are apparently more sensitive to amphotericin B than to the aromatic heptaenes vacidin A and candicidin D (Figs. 4 and 5 and Table 1).

Nystatin exhibits a particular mode of action as compared to both amphotericin B and aromatic heptaenes. The proton efflux obtained under its action is extremely slow in cholesterol-containing vesicles as compared to those containing ergosterol. Figs. 6A and 6B, where as in Fig. 2, the percentage of proton released is plotted versus time, show that with ergosterol containing vesicles (Fig. $6 \mathrm{~B}$ ) the proton release reaches its plateau (which again depends upon nystatin and sterol concentration) within about 20 minutes. This compares well with the time scale of the action of amphotericin B and aromatic heptaenes. Such is not the case with cholesterol-containing vesicles, especially at low nystatin concentration. The proton efflux develops very slowly, lasts several hours and finally reaches a plateau value comparable to those obtained in about 20 minutes with ergosterol-containing vesicles. As a matter of fact this extreme slowness makes it difficult to precisely measure this plateau value. However, with this peculiar feature of nystatin taken into account, its efficiency in cholesterol-containing vesicles, as measured by the percentage of proton which can be released at relatively low concentration is not very different from its efficiency in ergosterol-containing vesicles.

\section{Discussion}

Two main conclusions can be drawn from the results obtained on vesicles: first, the quantitative difference in the sensitivity of cholesterol and ergosterol-containing vesicles to the two aromatic heptaenes vacidin $\mathrm{A}$ and candicidin $\mathrm{D}$ is not very important, as shown in Table 1 for vesicles containing 10 mole per cent sterols. Second, both aromatic heptaenes appear to be slightly less active than the non-aromatic heptaene amphotericin B. Obviously, at least on the second point, there is a clear discrepancy between these results and those obtained on biological membranes.

However, in order to draw conclusions relevant to the understanding of the activity of polyene on biological membranes from data obtained on simple model systems such as sonicated purely lipidic vesicles, it appears necessary to closely consider the experimental conditions in which this activity is tested in both cases.

Aromatic heptaenes have been tested comparatively on yeast and red blood cells as representatives of ergosterol or cholesterol-containing membranes ${ }^{8,8,13)}$. At variance with amphotericin $\mathrm{B}$, vacidin A (or rather aureofacin, which is a mixture of aromatic heptaenes, but mainly vacidin $\mathrm{A}$ ) and candicidin D are more efficient on RBC than on yeast. 
Comparing these results with those on vesicles, it must be considered that yeast membranes contain about $20 \%$ ergosterol whereas RBC membranes contain more than $30 \%$ cholesterol. Therefore the comparison should be made with vesicles of the same sterol concentrations, since this parameter is very important: one can see that there is no real significant difference between the activity of both vacidin A and candicidin $\mathrm{D}$ on ergosterol and cholesterol-containing vesicles.

In biological membranes, aromatic heptaenes are $10 \sim 100$ times more active than amphotericin B. This difference has been found not only on RBC and yeast, but also on cell types such as the baby Hamster kidney cells $(\mathrm{BHK}-21)^{10)}$. It is mainly this fact which prompted the interest for the aromatic heptaenes. Such a large difference is absent in cholesterol or ergosterol-vesicles, in which the permeability is induced within about the same concentration range for aromatic and non-aromatic heptaenes.

In order to account for this obvious discrepancy, it seems necessary to consider closely the mechanism of action of these polyenes. The main information about the mechanism comes from the numerous studies on amphotericin B: nothing specific is known about aromatic heptaenes. However, since vacidin $A$ and candicidin $D$ are known to form intercationic selective pathways, it is reasonable to assume that their mechanisms of interaction with membrane is comparable to amphotericin B and that the molecular basis of their action is the formation of pores by polyene-sterol complexes including several molecules of each ${ }^{21)}$.

In the experiment reported here, the polyene concentrations studied range from $0.25 \sim 2.5 \times 10^{-3}$ $\mu$ mole $/ \mu$ mole lipids, that is about $0.5 \sim 5$ polyene molecules per vesicle: in other words, there are not enough polyene molecules to provide each vesicle with a pore. However as pointed out already by VAN HoOGEVEST and De KRUIJFF ${ }^{17}$, the reaction proceeds by rapid exchange of the polyene between the vesicles. Moreover it may be assumed, according to Gent and PRESTEGARD ${ }^{22)}$ that, due to the very small size of a sonicated vesicle, less than 1 millisecond is sufficient to equilibrate the internal and external medium, once the pore formed. Therefore upon addition of the polyene, as relatively concentrated solution in DMF, the proton flux is observed to be correlated with the dispersion of the polyene by exchange throughout the vesicle population. At low concentration, as the dispersion proceeds, the probability of pore formation, which demands a given number of molecules being present at a time in one vesicle, becomes vanishingly small and the proton flux stops. This can explain the fact that independent of time, the percentage of proton released (which in this hypothesis would correspond to the percentage of vesicle measured during this process) depends upon the number of polyene molecule per vesicles.

The situation in the biological membranes studied, appears to be very different: the polyene concentration necessary for $50 \%$ potassium leak, which is the most classical test for activity, is within about the same range as in vesicles, expressed in $\mu$ mole $/ \mu$ mole membrane lipids, that is about $10^{-3}$. However, this means that the number of polyene molecules per cell is orders of magnitude higher than in vesicles, and each cell has undoubtably enough polyene molecules to make many pores. Therefore, among different polyenes, the difference in activity has to be related to the stability of the pores formed, their life time and their intrinsic permeability properties.

On the basis of this reasoning, there is no discrepancy between the results obtained on cells and on vesicles since actually different parameters are tested.

In conclusion, the much greater potency as permeabilizing and cytotoxic agents of aromatic heptaenes as compared to non-aromatic heptaenes, appears to be explained in terms of the rate constant rather than in the order of the reaction of pore formation.

Acknowledgment

This work was partially supported by grant LA 219 C.N.R.S.

\section{References}

1) Hammond, S. M.: Biological activity of polyene antibiotics. Progr. Med. Chem. 14: 104 179, 1977 
2) Gale, E. F.: The release of potassium ions from Candida albicans in the presence of polyene antibiotics. J. Gen. Microbiol. 80: 451 465, 1974

3) Liras, P. \& J. O. LAmpen: Sequence of candicidin action on yeast cells. Biochim. Biophys. Acta 372 : $141 \sim 153,1974$

4) Borowski, E. \& B. Cybulska: Potassium death of Saccharomyces cerevisiae cells treated with N-succinylperimycin and the reversal of fungicidal action of the antibiotic by potassium ions. Nature 213: 1034 1035,1967

5) Cybulska, B. \& E. Borowski: Effect of N-succinyl-perimycin on yeast membrane permeability to monovalent cation. In "Systemic Fungicides". Lyr. \& Polter.-Akademie Verlag 1974: 83 92, 1974

6) Borowski, E.; B. Malewicz, B. Cybulska \& L. Falkowski: The interaction of polyene antibiotics with plasma membrane of Chlorella vulgaris. In "Systemic Fungicides". Lyr. \& Polter. -Akademie Verlag 1974: $93 \sim 100,1974$

7) Cybulska, B.; J. Mazerski, J. Zielinski, T. Ziminski \& E. Borowski: The role of structural factors in the modification of membrane permeability to external ions induced by polyene macrolide antibiotics. Drugs Exptl. Clin. Res. 6: 449 456, 1980

8) Kotler-Brajtburg, J.; G. Medoff, G. S. Kobayashi, S. Boggs, D. Schlessinger, R. C. Pandey \& K. L. RINEHART, Jr.: Classification of polyene antibiotics according to chemical structure and biological effect. Antimicr. Agents \& Chemoth. 15: 716 722, 1979

9) Brajtburg, J.; G. Medoff, G. S. Kobayashi, S. Elberg \& C. Finegold: Permeabilizing and hemolytic action of large and small polyene antibiotics in human erythrocytes. Antimicr. Agents \& Chemoth. 18: $586 \sim 592,1980$

10) Malewicz, B.; H. M. Jenkin \& E. Borowski: Dissociation between the induction of potassium efflux and cytostatic activity of polyene macrolide in mammalian cells. Antimicr. Agents \& Chemoth. 17: $699 \sim 706,1980$

11) Malewicz, B.; H. M. Jenkin \& E. Borowski: The repair of membrane alteration induced in baby hamster kidney cells by polyene macrolides antibiotics. Antimicr. Agents \& Chemoth. 1981 (in press).

12) Malewicz, B. \& E. Borowski: Energy dependence and reversibility of membrane alterations induced by polyene macrolide antibiotics in Chlorella vulgaris. Nature 281: 80 82, 1979

13) Cybulska, B.; E. Jakobs, L. Falkowski \& E. Borowski: Structure-selective toxicity relationship in polyene macrolide antifungal antibiotics. In "Systemic Fungicides". Lyr. \& Polter. -Akademie Verlag 1974: $77 \sim 81,1974$

14) Kasumov, K. M. \& E. A. Liberman: Ionic permeability of bimolecular membranes in the presence of polyenic antibiotics. Biofizika 18: 64 271, 1973

15) Patel, K. M. \& J. T. Sparrow: Rapid, large-scale purification of crude egg phospholipids using radially compressed silica gel columns. J. Chromatogr. 150: 542 547, 1979

16) Prigent, Y.; S. Trandinh \& C. M. Gary-Bobo: Proton gradient across membranes of lecithin vesicles as measured by ${ }^{31} \mathrm{P}$ NMR. Asymmetrical behaviour of internal and external layer. Biochem. Biophys. Res. Commun. 95: 1218 1223, 1980

17) Van Hoogevest, P. \& B. De KruijfF: Effect of amphotericin B on cholesterol containing liposomes of egg-phosphatidylcholine: A refinement of the model for the formation of pores by amphotericin B in membranes. Biochim. Biophys. Acta 511: $397 \sim 407,1978$

18) Mason, J. T. \& C. Huang: Hydrodynamic analysis of egg phosphatidyl-choline vesicles. Ann. N. Y. Acad. Sci. 1978: 29 49, 1978

19) De Kruijff, B.; W. J. Gerrisen, A. Oerlemans, R. A. Demel \& L. L. M. Van Deenen: Polyene antibioticsterol interaction in membranes of Acholeplasma laidlawii cells and lecithin liposomes. I. Specificity of the membrane permeability changes induced by the polyene antibiotics. Biochim. Biophys. Acta 339: $30 \sim 43,1974$

20) Teerlink, T.; B. De Kruijff \& R. A. Demel: The action of pimaricin; etruscomycin and pimaricin B on liposomes with varying sterol content. Biochim. Biophys. Acta 599: 484 492, 1980

21) De KKruijff, B. \& R. A. Demel: Polyene antibiotics-sterol interactions in membranes of Acholeplasma laidlawii cells and lecithin liposomes. III. Molecular structure of the polyene antibiotic-cholesterol complexes. Biochim. Biophys. Acta 399: 57 70, 1974

22) Gent, M. P. N. \& J. H. Prestegard: Interaction of polyene antibiotics with lipid bilayer vesicles containing cholesterol. Biochim. Biophys. Acta 426: 17 30, 1976 